\title{
4-5-year-old children adapt to the reliability of conflicting sources of information to learn novel words
}

\author{
Marion Beretti ${ }^{1}$, Naomi Havron*1, Anne Christophe ${ }^{1}$ \\ ${ }^{1}$ Laboratoire de sciences cognitives et de psycholinguistique, ENS, EHESS, CNRS, PSL \\ University \\ * Joint first authors
}

This is a preprint paper of a paper accepted to publication in Journal of Experimental Child Psychology

To be cited as: Beretti, M., Havron, N., \& Christophe, A. (in press). 4-5-year-old children adapt to the reliability of conflicting sources of information to learn novel words. Journal of Experimental Child Psychology.

Conflict of Interest Statement: The authors declare no conflict of interests

Acknowledgments: This work was supported by the French Agence Nationale de la Recherche (grants ANR-13-APPR-0012 LangLearn, ANR-17-CE28-0007-01 LangAge, and ANR-17EURE-0017 FrontCog); postdoctoral grants to Naomi Havron from the French Embassy in Israel and the Victor Smorgon Charitable Fund; a CIF grant to Marion Beretti from the Fongecif Ile De France. We thank the school and children for their participation.

Keywords: cue integration, adaptation, reliability, conflict resolution, language acquisition, syntactic bootstrapping

Corresponding Authors:

Marion Beretti (beretti.marion@gmail.com) \& Naomi Havron (naomi.havron@ mail.huji.ac.il) 


\section{Research highlights}

- Children and adults adjust to reliability of cues to disambiguate sentences.

- They use their adapted expectations when homophones need to be learned.

- The effect was stronger in adults. 
A central challenge in language acquisition is the integration of multiple sources of information, potentially in conflict, to acquire new knowledge and adjust current linguistic representations. One way to accomplish this is to assign more weight to more reliable sources of information in context. We test the hypothesis that children adjust the weight of different sources of information during learning, considering two specific sources of information: their knowledge of the meaning of familiar words (semantics) and their familiarity with syntax. We varied the reliability of these sources of information through an induction phase (reliable-syntax or reliable-semantics). At test, French 4-5-year-old children and adults listened to sentences where information provided by these two cues conflicted, and were asked to choose between two videos that illustrate the sentence. One video presented the reasonable choice if the sentence is assumed to be syntactically correct, but familiar words refer to novel things (e.g., une mange "an eats" describes a novel object). The other video was the reasonable choice if the sentence is assumed to be syntactically incorrect and familiar words' meaning is preserved (e.g., "an eats" describes a girl eating, and should have actually been "she eats"). As predicted, the proportion of syntactic choices (e.g., interpreting mange - "eats", as a novel noun) was found to be higher in the reliable-syntax condition than in the reliable-semantics condition, showing that children and adults can adapt their expectations to the reliability of sources of information. 


\section{Introduction}

Children's knowledge of language is imperfect. As they learn to process new sentences, they might encounter words they do not know. To learn the meaning of these novel words, children and infants use, among other things, their knowledge of syntax to constrain the space of possible meanings. This is called syntactic bootstrapping (e.g., Bernal, Lidz, Millotte, \& Christophe, 2007; Gleitman, 1990). Thus, if they hear "she is daxing", children who do not know what "dax" means can at least infer that it is a verb (and probably refers to an action or state). If they hear "she is daxing that girl" they also know that this is a transitive verb (and probably refer to a causative action; Arunachalam, 2016). But as they learn these novel words by relying on syntax, children must also learn about syntax from words they know (Christophe, Dautriche, de Carvalho, \& Brusini, 2016). For example, in a recent study, familiar words were used to induce children to expect either a noun or a verb following a specific syntactic context (that could be followed by either nouns or verbs). Thus, after hearing four familiar verbs in this context, children were more likely to infer that a novel word appearing in this context referred to an action, than children who heard four familiar nouns in this context (Havron, de Carvalho, Fiévet, \& Christophe, 2019).

Some real-life situations may offer a challenge for a child who is still learning about syntax while acquiring novel words. Take for example a study conducted with adults. In this study, adults learned a new construction "this X needs Xed", which is used in the Northern Midlands dialect of American English (e.g., "this cake needs baked"). Adults learned that in the dialect they were reading, "this cake needs baked" means something like "this cake needs baking", and started anticipating this construction (Kaschak \& Glenberg, 2004). Imagine a child who hears a sentence such as "this cake needs baked". How does the child know whether they should learn a new construction, or whether the word "baked" means something like "decorations" or some ingredient that is lacking in the cake? They might always rely on one source of information and 
prioritize it over the others (e.g., always assume that their current representation of syntax is more reliable than their current lexicon), or they might dynamically decide which cue to use in each specific context.

One reasonable way to dynamically change their reliance on different cues is to assign more weight to the more reliable source of information (Gibson, Bergen, \& Piantadosi, 2013). This idea is expressed by Gibson and colleagues' use of the concept of the noisy channel. According to their noisy-channel account, sentence comprehension operates by rationally combining prior information about what constitutes a plausible utterance with a model of the reliability of different sources of information (i.e., the level and type of noise in the environment). This idea can be viewed through the lens of Bayesian probability: a sentence is interpreted by taking into account prior expectations, and combining these with novel evidence, to create a new distribution of expectations (the posterior). The decision whether and what to change from the old to the new distribution is a meta-Bayesian decision. We decide which set of expectations led to our error in prediction by estimating which source of evidence is more likely to have been noisy in context. In other words, changing the probability of one piece of information affects the relative weight of all others - inserting noise in one channel makes the other channels more reliable and a stronger source of evidence to rely on. Gibson and colleagues (2013) tested this weight adaptation by having adults listen to a strange sentence such as "The ball kicked the girl" and answer a yes-or-no comprehension question (e.g. "Did the girl kick something/someone?"). In one condition, syntax errors were inserted into filler items (e.g., function words were deleted or added) in order to reduce the likelihood of the literal meaning by rendering syntax less reliable; in the other condition, semantically implausible items were inserted into the fillers to limit semantic reliability, thereby privile ging a literal reading of the sentence. When the syntax of the speaker appeared noisy overall, adults preferred to mentally correct the test sentence, and assume that the speaker meant that the girl kicked the ball but 
made a syntactic error. Thus, they chose the most plausible meaning (e.g. answer "yes" to "Did the girl kick something/someone?"). When implausible items were common, adults preferred to preserve the literal meaning (e.g. answer "no" to "Did the girl kick something/someone?"). Thus, the noisy channel model explains how language users integrate multiple sources of information by prioritizing each one depending on its own reliability in context.

A similar intuition had previously been incorporated into language acquisition research in the context of the Competition Model (Bates \& MacWhinney, 1989). In this model, language acquisition is described as cue-driven distributional analysis of the linguistic input. The model makes the prediction that children growing up learning different languages would prioritize different cues depending on their saliency, reliability and differential reliability in cases of conflict. Thus, for example, Chan, Lieven, \& Tomasello (2009) found that when they presented children with sentences where animacy cues conflicted with word-order cues, Englishacquiring children showed earlier and stronger reliance on word order than children acquiring both German and Cantonese, where word-order cues are less reliable. Though different languages constitute large-scale natural experiments, such differences could also be due to certain confounds. Thus, it is important to also explore these ideas in experimental settings. Moreover, language-comparative studies can show how children learn through the stable and established distribution of cues in their language, but they cannot test whether and how children flexibly adapt to context, and to rapid changes and variation in distribution and reliability of cues.

A recent study tested these predictions experimentally, by looking at how children rapidly integrate different source of noise in context, in a design similar to Gibson and colleagues' study (2013). In this study by Yurovsky, Case, \& Frank (2017), 4-5-year-old children listened to ambiguous sentences, where the literal information and the semantically plausible information were in conflict (e.g., "I had carrots and bees for dinner"). The phonetic proximity 
between the presented word ("bees") and the most plausible word for this context ("peas"), made both interpretations possible. An induction phase manipulated the reliability of these two sources of information, phonetic and semantic. In one condition, the filler trials increased the ambient noise and thus decreased the reliability of the phonetic information in context (i.e., increasing the chances that the speaker likely meant "peas" but they had misheard them on account of the noise). In the second condition the researchers inserted implausible filler sentences (e.g., "my cat has three little hammers" with a choice between an image of a cat with hammers or a cat with kittens) to decrease the reliability of the semantic information, so that children would assume that the speaker indeed meant the strange literal meaning also in test sentences (i.e., that they ate bees). Children were not given feedback about whether their answer on filler (or test) trials was correct. On test trials, the child had to choose between two images representing these two interpretations. Indeed, children in the phonetically-noisy condition preferred the semantically plausible choice (assuming the speaker had meant the plausible alternative "peas"); but children who were exposed to a speaker who produced semantically implausible utterances were more likely to adopt the literal interpretation (assuming the speaker had really meant "bees"). These results seem to indicate that children's preference for one source of information over the other, in comprehension, is dependent on the reliability of each cue in context rather than on a default weighting scheme (with phonetics always weighted higher than semantic plausibility, or vice-versa). In the current paper, we investigate whether these results also apply to a situation central in language learning, when children are learning new words or building syntactic representations, rather than processing familiar sentences.

As mentioned above, for children, different sources of information which are involved in language acquisition are also in the process of being learnt. Two prominent sources of information which have been discussed in the literature as playing an important role in language learning are semantic and syntactic information. Some have proposed a default prioritization of 
bottom-up lexical cues compared to syntactic ones, which would be involved only when semantic cues are insufficient or ambiguous (e.g., Federmeier, 2007). Other researchers side with prediction-error models, where the weight of each source varies depending on its reliability in the context (e.g., Rabagliati, Pylkkänen, \& Marcus, 2012). There is currently little evidence to discriminate between these two suggestions in the literature on adults and young children (as discussed by Federmeier, 2007). It has, however, been shown that children are capable of using the reliability of the syntactic source of information, or of the semantic source of information, if each one is tested separately. For example, when comparing an induction condition where a syntactic cue is reliable, to another where the same cue is not, children can stop using syntactic cues for prediction (van Heugten, \& Christophe, 2014). Toddlers can even ignore the meaning of a familiar word, to learn a novel meaning for it (therefore creating a homophone), in a case in which the familiar meaning is inappropriate. For example, toddlers accept that "an eats " could be the name of a novel animal (see Dautriche, Fibla, Fiévet, \& Christophe, 2018). This resembles real-life situations in French and other languages. For example, in French, in the sentence elle sourit, "she is smiling", sourit is a homophone of the word souris, "mouse". If children do not know the word "smiling", only "mouse", but they do know that elle is usually followed by a verb, and never by a noun - they should understand that sourit in this context has to be a verb that they do not know, and create a homophone.

The goal of the current study is to understand if children are able to learn novel meanings and adapt their semantic and syntactic expectations, when the two sources of information (semantics and syntax) are pitted one against the other. Previous studies have never put these two cues in conflict - children would ignore syntax when it was unreliable, or ignore the familiar word meaning if it was unlikely, but never did they have syntax provide one answer and the lexicon a different one. When faced with a conflict, if children adapt to the reliability 
of different cues in context, this would support a noisy channel model of word learning, whereby children can adjust the weight of different cues in context.

We follow a paradigm similar to the one used in Yurovsky et al. (2017) to test the noisy channel hypothesis in language learning. To examine how children adapt the weight of their prior sources of knowledge (semantics or syntax), we compare two conditions, which vary in terms of which source of information is reliable. Children are presented with an induction phase, where either syntax is reliable (reliable-syntax condition) or semantics is reliable (reliablesemantics condition). In both conditions, children hear the same sentence (e.g., elle pomme “she apple(s)"). In the reliable-syntax condition, children see a novel action or object, which does not have a name in French, and are thus led to believe that the speaker is using a familiar word-form to refer to something the participant has not seen before (in the example pomme is a novel verb, that happens to sound like the noun pomme but has nothing to do with apples - a homophone). In the reliable-semantics condition children hear the same sentence. Now there is no novel action or object, but instead the person seems to be using syntax incorrectly. In the example above, there would be a video of a girl holding an apple and performing no action with it, and the speaker can then be assumed to have made a syntactic error, and to have meant "it is an apple" and not "she apple(s)". Thus, in the induction phase, the cues are not in conflict, since the presented videos are compatible with only one interpretation of the sentence.

In the test phase, participants hear a short sentence composed of a syntactic and a semantic cue that are in conflict (e.g., elle balle - "she ball(s)"), and they have to choose between two videos corresponding either to the syntactic cue (a girl performing a novel action) or to the semantic cue (a ball). When you hear an utterance such as "she ball(s)", if you think that this person's syntax is impeccable, but that she tends to introduce novel words, then you will be tempted to interpret "ball" as a verb you do not know yet, a homophone of the noun "ball". In that case, the correct referent of the utterance would be the girl performing the novel action. In 
contrast, if you think that this person's syntax is uncertain (because she has been employing familiar words in incorrect syntactic contexts before), while her use of familiar words is always perfectly appropriate, then you will tend to rely on your recognition of the content word "ball". You would deprioritize its syntactic context ("she"), which would lead you to choose the video of the girl holding a ball as the correct referent of the utterance. Thus, even though the test sentences have the same form as the induction sentences, the fact that a choice is offered between two reasonable options (the syntactic one and the semantic one) makes the sentences ambiguous, and puts the cues in conflict. We collected two measures: A pointing response representing participants' final decision, and a gaze measure measuring participants online processing of the sentence. Some of the previous studies we draw on (Dautriche, et al., 2018; Havron et al., 2019) used eyetracking, while others used only pointing (Yurovsky et al., 2016). Using an eyetracking measure provides a window into participants' sentence processing in real time, as they switch their gaze between the two videos as the sentence unfolds.

We test whether 4-5-year-old children are capable of using the reliability of one source of information compared to the other in order to interpret these sentences, or whether they preserve a default weight for each source of information, regardless of its reliability (either always follow the syntax or always follow the semantics). If participants have adapted to the reliability of the sources of the information, they will choose the syntactic choice more often in the reliablesyntax condition than in the reliable-semantics condition (either explicitly by pointing, or implicitly by looking at the video). If participants in the reliable-syntax condition choose the syntactic choice more than participants in the reliable-semantics condition, they will be shown not only to be able to take into account the reliability of a source of information for comprehension, but also, in the reliable-syntax condition, to rely on the prioritized source of information to learn novel words (even when this entails the creation of homophones). We also tested a group of adult participants, in light of previous experiments showing stronger 
adaptation effects in adults (e.g., Havron et al., 2019), and in order to see whether adults have a different bias (either for syntax or semantics) than children. Differences between children and adults may stem from task demands. Children have less developed cognitive skills such as perception, memory, and executive functions, which may render them less likely to integrate the information present in the experiment, simply because of the greater burden on their cognitive resources. However, differences between children and adults may also stem from true differences in processing in real life. The costs of processing a given linguistic structure might weigh more heavily for children, and thus attenuate the impact of reliability information on language learning. Indeed, Bates and MacWhinney (1989) suggest that it is around the age of six that children start assigning less weight to semantic factors and start relying more reliably on morphosyntax for sentence interpretation.

\section{Method}

This study was approved by the Comité d'éthique de la recherche en santé (CERES, IRB: 20140100001072). All data and materials have been made publicly available via the Open Science Framework: https://osf.io/fsb92/?view_only=000d7f4b94d64428a29aff67dd9ce583. The design and analysis plans were preregistered at the OSF and can also be accessed with this same project.

\section{Experimental Design}

The experiment is based on exposure to ungrammatical sentences, like elle balle - "she ball(s)" or une parle - "a speak". We use induction items to weaken participants' confidence either in the way the speaker is using either syntax or in the way she is using semantics, depending on the experimental condition (see Fig. 1). Thus, the same sentence is interpreted as being syntactically correct and introducing a homophone by one group of participants, and as being syntactically incorrect by the other group of participants. At test, we present sentences of the 
same type, but now place the cues in conflict by showing two videos: one illustrates the semantic choice (where the known lexical item "ball" is shown in the video), and the other the syntactic choice (where a novel action provides a good fit for the novel content word). We ask participants to point to the video corresponding to the sentence they heard (see bottom of Fig.

1).

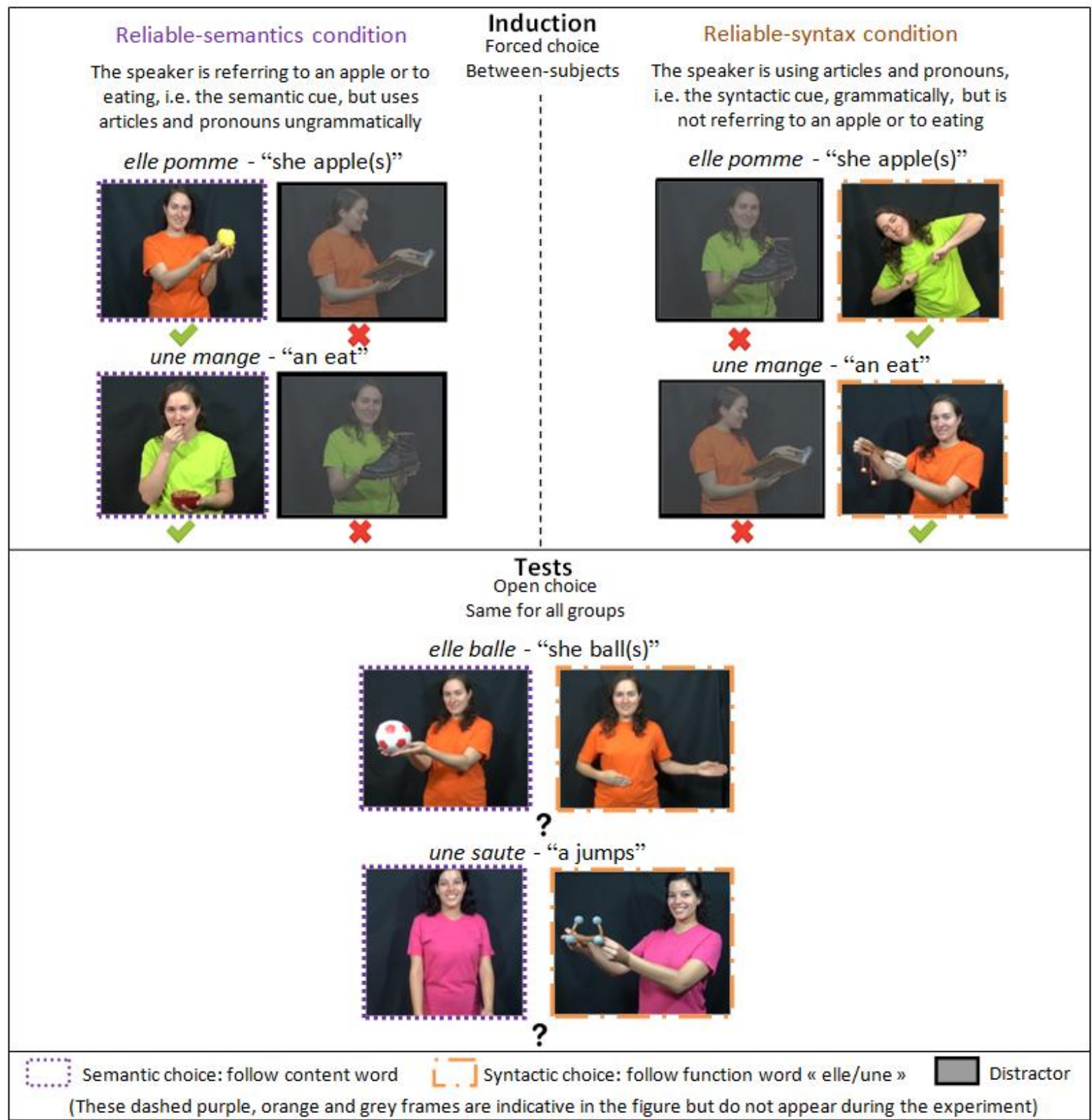

Figure 1. Experimental design: induction phase ( 2 conditions) and test phase

Thus, in the reliable-semantics condition, induction items encourage participants to follow the semantics of the content word, and to ignore the preceding function word. For example, when 
hearing elle pomme - "she apple(s)", they are given a choice between an apple and a distractor, a familiar action like reading, which is not a good candidate for the meaning of a novel verb pommer - "to apple", since the action of reading already has a label in French (the mutual exclusivity principle, Markman \& Wachtel, 1988). In this example, as in all our items, we make sure that the familiar noun ("apple") is not a good candidate for a verb interpretation (elle pomme, "to apple" means performing some action with an apple) because the object is not involved in any action (e.g., the girl is not doing anything with the apple that could be described as "appling"). In the reliable-syntax condition, participants are encouraged to trust the function word to assign a new syntactic category to the following content word, while ignoring the fact that the form of the content word already has a meaning in French. For example, when hearing elle pomme - "she apple(s)", they are faced with a novel action (a good candidate for novel verb pommer - "to apple"), and a shoe (which has nothing to do with an apple) as a distractor. In this condition, participants are encouraged to create a new homophone for the familiar word (pomme - "apple") - a word that would come from a different syntactic category (here, a verb). Infants as young as 20 months are able to create homophones (Dautriche et al., 2018) when taught a novel word from a different syntactic category (e.g., they can learn that the novel noun "a give" represents a furry animal, even though they already know the verb "to give"). Note that in Dautriche and colleagues' experiment, there was only a furry animal present as a potential candidate for "a give" - there was never also someone giving something, and thus no conflict, as opposed to our test phase.

We use a between-subject design, with each participant randomly assigned to one of these conditions. Each participant hears examples of both nouns and verbs, in a counterbalanced manner as described in more details in the procedure section below (e.g. elle balle - "she ball(s)" for verb syntactic cue and noun semantic cue; une parle - "a speak" for noun syntactic cue and verb semantic cue). 


\section{Participants}

Our final sample consisted of 57 middle to high SES 4-5-year-old children (25 girls, 32 boys, $\mathrm{M}$ age $=57.36$ months, range $=48.20-66.61$ months), and 51 adults (18 women, 33 men) who were randomly assigned to the two conditions. Children were tested either in their kindergarten or in our laboratory. Adults were tested either in their workplace or in our laboratory. Eyetracking data was filtered with the following criteria: we excluded trials with more than $25 \%$ of missing data, and participants with more than $50 \%$ of excluded trials (10 children and 14 adults excluded ${ }^{1}$ ). We kept pointing data for these trials/participants leaving us with 29 children in the reliable-semantics condition (24 also have eyetracking data) and 28 in the reliable-syntax condition (23 also have eyetracking data); and 26 adults in the reliablesemantics condition (15 also have eyetracking data) and 25 in the reliable-syntax condition (22 also have eyetracking data). Four additional children were tested but their data was not included in the final analyses, because they were bilingual (2), fussy (1) or had a psychological disorder (1). Data of one additional adult was excluded because he was bilingual. We preregistered that we would test at least 24 participants in each group, the exact number depending on the number of consent forms obtained by the parents. With 24 children in each group, we have $80 \%$ power to detect an effect of the size $d=0.75$, while the effect size for adaptation in grammatical contexts (and younger children) in Havron et al. (2019) was 0.94. Our final sample size allows us to detect effect sizes as small as 0.65 with $80 \%$ power, but with a moderate effect size, such as 0.4 , we would only have about $60 \%$ power.

\section{Materials}

Sentences and videos. All sentences are composed of two words presenting a conflict between syntax and semantics (e.g. elle balle - "she ball(s)" or une parle - "a speak"): (1) A

\footnotetext{
${ }^{1}$ This is quite a high exclusion rate for adult participants. In addition to regular factors contributing to low quality data from adults (e.g., glasses, eyeliner) - it might have stemmed from a combination of two factors: most adults were tested outside the lab, and the experimenter testing them was relatively inexperienced.
} 
function word for the syntactic cue (i.e. article une - "a" for nouns or pronoun elle - "she" for verbs). (2) A familiar content word for the semantic cue.

Stimuli were videos, always paired to present an action and an object on opposite sides of the screen. Objects and persons all had feminine gender to avoid the additional complexity of grammatical gender. For actions, the video presents a woman performing the action; for objects, the same woman is seen holding an object in her hands while looking at it and at the camera alternately - to encourage participants to look at it instead of at herself. All target actions corresponded to intransitive verbs and all objects were inanimate. Both verbs and nouns were selected from Lexique.org (New, Pallier, Ferrand, and Matos, 2001) and Wordbank (Frank, Braginsky, Yurovsky, \& Marchman, 2017) with the criteria that they were well-known to young children, even those much younger than $4-5$, and were performable on a video for verbs, and small enough to be hand-held, and recognizable on a video, for nouns. Since the number of available items was restricted, we sometimes used actions which included objects (e.g. reading, which included a book), but only for distractors. Labels associated with novel words had to be plausible homophones. For this reason, we chose monosyllabic words belonging to a dense phonological neighborhood (Piantadosi, Tily, \& Gibson, 2012). To contrast the videos which we wanted the children to select in the induction phase, distractor videos were not too close to the original meaning of the word, in order to avoid a possible polysemy with the distractor video. For example, if the distractor for the sentence elle pomme - "she apple(s)", had been a woman eating, elle pomme could be interpreted as meaning "eating an apple", such that "to apple" and "an apple" would share a core meaning, a case of polysemy. We therefore presented a girl reading as the distractor, so that it would be unreasonable to suspect that elle pomme means "she reads". For each video, target words always appeared in the same time window: first onset around $2.4 \mathrm{~s}$ and second around $7.5 \mathrm{~s}$. 
A typical trial. For each item, each of the videos is first presented alone for eight seconds, then a fixation point appears and stays on the screen for 500ms after the child fixates on it (as automatically detected by the eyetracker). Then both videos are presented together for ten more seconds and the critical sentence is played (see Fig. 2). The target side is random, but with an equal total number of left and right targets for a given participant. At the end of the trial, the experimenter asks the participant to point at the video corresponding to the sentence. Pointing is recorded by the experimenter on the computer, and gaze is recorded for the whole duration of the trial. In one-video-induction trials, only one video is displayed on the screen with no distractor. The inclusion of one-video items was done to shorten the overall length of the experiment. In these items, even more than in two-video items, participants would draw the correct conclusion about the meaning of the sentences (depending on the condition they are in, see Appendix for the items), because no distractor is available. No feedback is given to the participants to validate their interpretation after their choice, in any item, induction or test, as was the case in other similar previous studies (Havron et al, 2019; Yurovsky et al, 2016 and Dautriche et al, 2018).

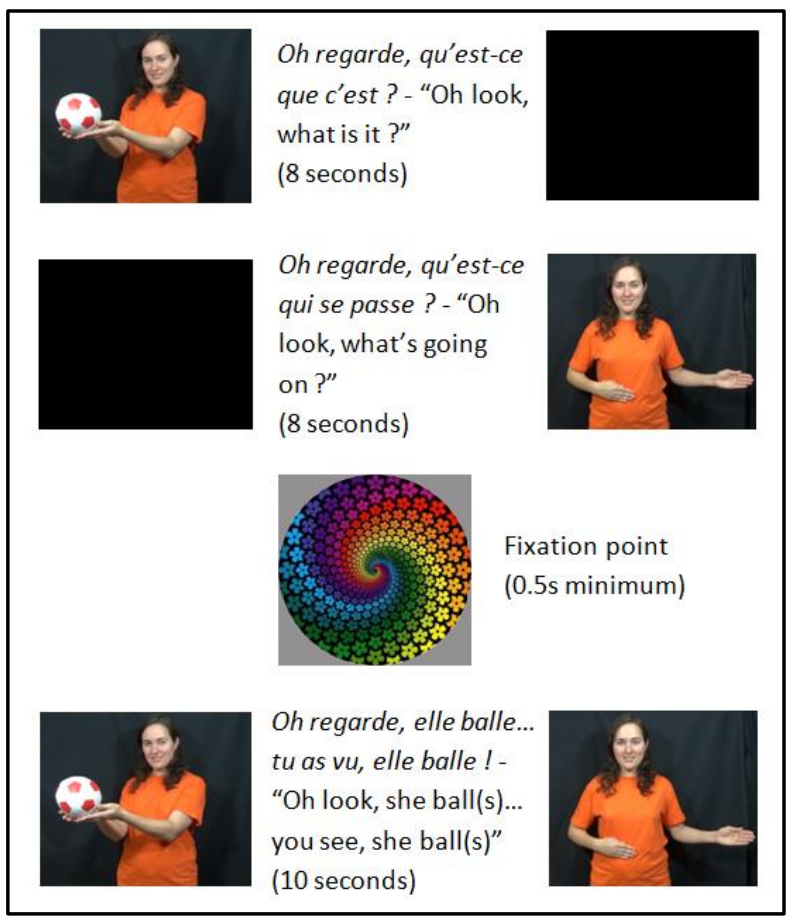

Figure 2. Trial sequence (for each item, example for a test item) 


\section{Procedure}

Global organization. The experiment was launched from Matlab (Version R2011b 7.13.0.564, MathWorks, Inc., 2011) on a portable computer, and videos were displayed on a 27 ' screen placed 70cm from the participant. An Eyelink II eyetracker (camera: EyeLink CL Version 1.4 Sensor BIB) was positioned under the screen and connected to the computer to record participants' gaze during the whole task.

Following a calibration phase, each participant watched a series of 14 video items (see Fig. 3), distributed in the following manner (phase transitions are transparent to the participants): Two warm-up items which always showed an object video + an action video. These items were meant to introduce participants to the task, and make sure that they understand that they need to choose the video "the lady was talking about". For example, participants would see a girl eating and a girl holding a ball, and hear the sentence c'est une balle, "that's a balle", and were asked to choose the video the lady was talking about (the one with the girl holding a ball). The warm up phase was followed by four one-video induction items (presented with only one video, without a distractor, to reduce the length of the experiment and reinforce learning). Then, children saw two induction items (pairs of target + distractor), followed by two conflict test items (pairs of semantic vs. syntactic interpretations), followed by another pair of induction items and another pair of conflict-test trials. Conflict-test items were interspersed within the induction items in order to reinforce the induction before each test. Each of the last four miniblocks (first two-video induction block, first test block, second two-video induction block, second test block) contained exactly two items, always one verb and one noun. The different verbs and nouns were randomized between the first and the second block of the same kind (between the two induction mini-blocks, and between the two test mini-blocks). The order of appearance inside one mini-block (first the verb and then the noun, or first the noun and then the verb) was also randomized. For each item, participants were asked to point to the video they 
thought corresponded best to the sentence they heard. The dependent measures were pointing and proportion of looks towards the syntactic choice. If participants are adapting to the reliability of the speaker, then we expect children from the reliable-syntax condition to point more and look more at the syntactic choice, the homophone, than participants in the reliablesemantics condition.

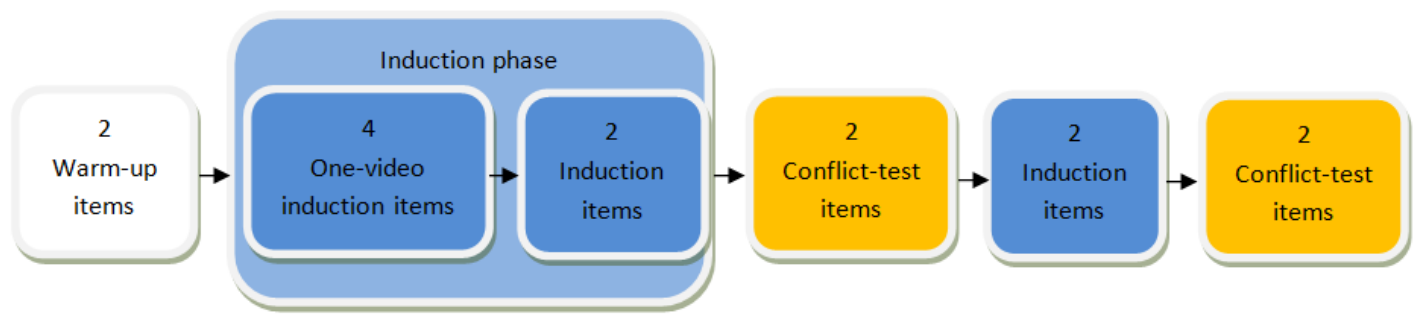

Figure 3. Task phases for each participant

Only one-video induction items and induction items change between conditions, warm-up and test items are the same for all participants. All participants in a given condition saw exactly the same items, the order of which was randomized within each phase.

Additional items. Our test items can tell us whether participants can adapt to the reliability of cues to change their syntactic expectations, or learn something about semantics - but in the former case, they do not tell us whether syntactic expectations were abandoned or adjusted. To further probe participants' ability to learn, and explore whether they also learn something about syntax, we added two novel-word trials at the end of the experiment. The idea was to examine what syntactic knowledge the participants had created or retained at the end of the task (their initial knowledge, or a novel adapted one) by presenting sentences like elle dase - "she dase(s)" or une nuve - "a nuve". Since these are novel words, that could be interpreted either as a noun or a verb, participants who have kept their prior predictions should expect dase, which is preceded by a pronoun, to be a novel verb and nuve, which is preceded by a determiner, to be a novel noun. If, however, participants have created new predictions based on the distribution of words in the induction phase, then we expect only the reliable-syntax group but not the 
reliable-semantics group to entertain these interpretations. These two items were tacked at the end of the experiment, so that they did not disrupt the main experiment: as there are only two items, and they are presented when the children have likely already tired, they are underpowered to draw firm conclusions, and we only report the results in the supplementary materials.

\section{Statistical Analysis}

Statistical analyses were conducted with R software (RStudio 1.0.136), using packages lme4 (Bates, Maechler, Bolker, \& Walker, 2015) and eyetrackingR (Dink \& Ferguson, 2016), and plotted with ggplot2 (Wickham, 2016). Only test trials were considered for analysis. If participants are able to adapt to the reliability of a source of information in order to interpret sentences, they will choose the syntactic choice more in the reliable-syntax condition than in the reliable-semantics condition. We also examine whether participants preferred overall the semantic or syntactic choice by looking at the intercept of the analyses for pointing, and performing a one-sample t-test for proportion of looks.

We ran mixed-effects logistic regression analyses ${ }^{2}$ on pointing and overall proportion of looks (Jaeger, 2008). The dependent variables were the proportion of pointing or looks towards the syntactic choice (from after the end of the first time the participants hear the sentence until the end of the trial). The choice corresponding to the elle/une - "she/a" cue (e.g. the girl performing the novel action for elle pomme - "she apple(s)"), both for pointing and eyetracking data is designated as the syntactic choice. Syntactic and semantic choices are complementary. In both analyses we had condition (sum coded), age (children vs adults, sum coded) and an interaction between age and condition as independent variables. The models included random intercepts for participant and item, and the model for the eyetracking data also included a by-

\footnotetext{
${ }^{2}$ For pointing only, instead of glmer that did not converge (perhaps because of the fact that all participants in the semantic condition chose the semantic choice), mixed-effect regression models have been run using bglmer, with default parameters (Bayesian layer adding a weak informative prior on the fixed-effects parameters, as suggested in Gelman, Jakulin, Pittau, \& Su, 2008).
} 
item random slope for condition - the maximal random effect structure that allowed the models to converge.

To better understand the online processes, we also ran a time-course analysis by conducting a cluster-based permutation analysis on mean proportion of looks towards syntactic choice in each 20 milliseconds time bin as a function of time (our eyetracker samples gaze location for each 2 milliseconds, which we then bin to 20 milliseconds bins). This analysis determines whether there are time windows where a difference between the two conditions is significant. For each time sample, the analysis runs a t-test testing for the effect of condition (on the arc-sin transformed proportion of looks toward the syntactic choice video). Adjacent time points with a $\mathrm{t}$-value greater than a predefined threshold $(\mathrm{t}=1.5)$ are grouped together into a cluster. The size of the cluster is defined as the sum of the $t$ values at each time point within the cluster. To compute the probability of observing a cluster of that size by chance, the data is reshuffled randomly between the two conditions a thousand times, and the same cluster-finding procedure is applied to each of the 1000 simulations. The $\mathrm{p}$ value of the effect of condition for a cluster is calculated as the proportion of simulations that produce a cluster larger than the cluster from the original data. Thus, a cluster is considered significant if it is larger than $95 \%$ of the clusters generated by chance.

\section{Results}

\section{Pointing}

As hypothesized, we found a main effect of condition $(\beta=2.22, \mathrm{SE}=.56, \mathrm{z}=3.97, \mathrm{p}<.001)$ : participants in the reliable-syntax condition pointed more often towards the syntactic choice than participants in the reliable-semantics condition, suggesting that participants used the respective reliability of syntax and semantics as a cue to weigh the different sources of information. The main effect of age was marginally significant $(\beta=-1.06, \mathrm{SE}=.55, \mathrm{z}=-1.92$, $\mathrm{p}=.055)$ with adults selecting the syntactic choice slightly less often than children (adults: 
mean $29.41 \%(\mathrm{SD}=42.04 \%)$, children: mean $32.89 \%(\mathrm{SD}=30.03 \%))$. There was a significant interaction between age and condition $(\beta=1.65, \mathrm{SE}=.55, \mathrm{z}=2.99, \mathrm{p}=.003)$ : the induction had a stronger effect for adults, who chose the reliable video more often than children, in both conditions (semantics and syntax). In the reliable-semantics condition, semantics seem particularly strong, since adults made this choice on every trial (see Fig. 4). When running the same model separately for children and adults, the effect of condition is significant for both groups (children: $\beta=.46, \mathrm{SE}=.22, \mathrm{z}=2.09, \mathrm{p}=.04$; adults: $\beta=4.07, \mathrm{SE}=.93, \mathrm{z}=4.39, \mathrm{p}<$ $.0001)$. See Figure 4.

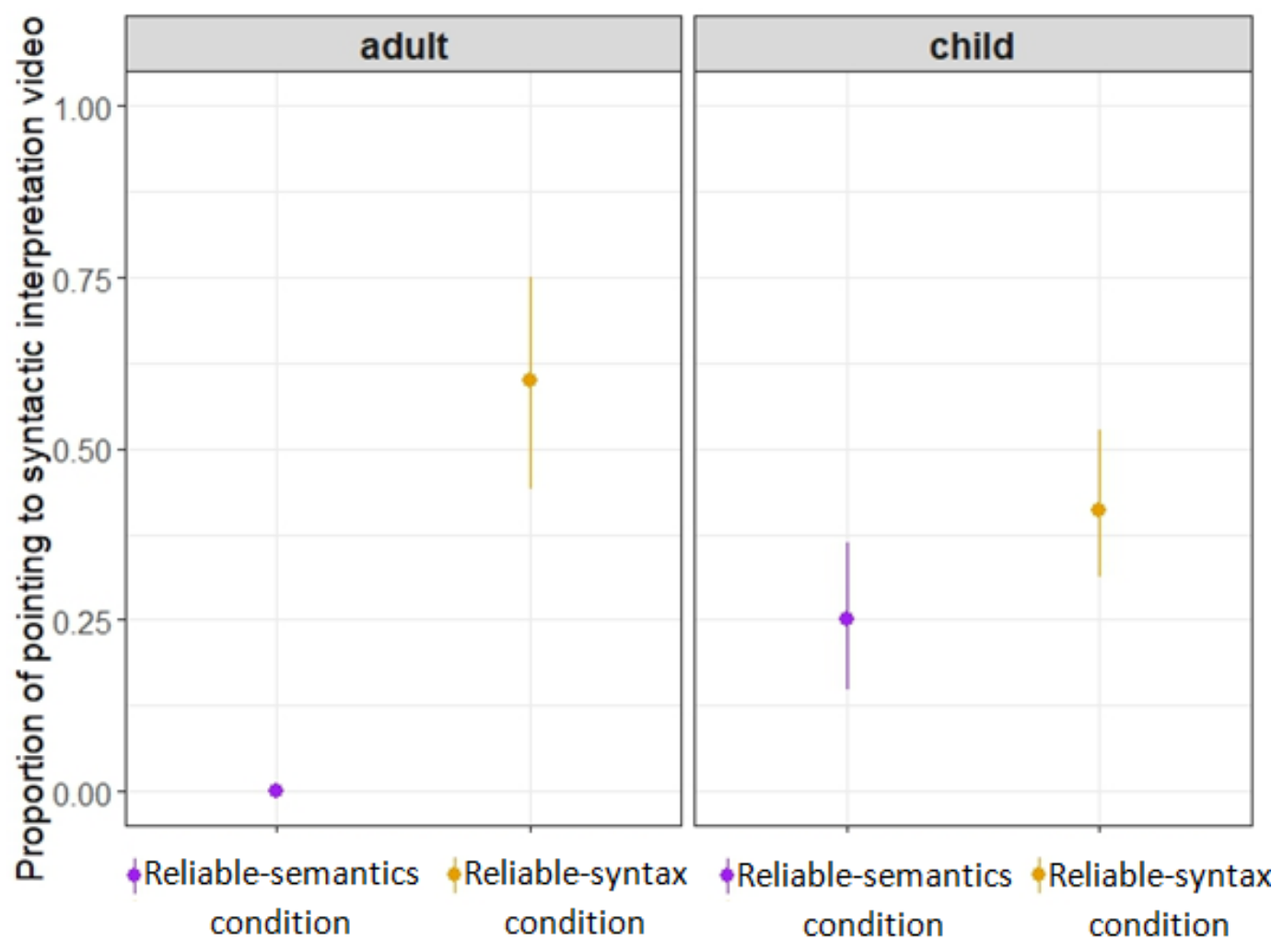

Figure 4. Proportion of pointing towards the syntactic choice, per condition (reliable-syntax vs reliable-semantics), for adults and children. The dot is the average for each condition and group, lines represent standard errors from bootstrapping as calculated by ggplot 2 . For both children and adults, there are more syntactic choices in the reliable-syntax condition. 
To test for a possible bias towards the semantic vs syntactic choice, we compared the proportion of pointing responses to chance $(50 \%)$, in all conditions taken together. A preference is observed towards semantics (the intercept of the mixed-effect model is negative and significant: $\beta=-1.71, \mathrm{SE}=0.39, \mathrm{z}=-4.42, \mathrm{p}<.00001$, see Fig. 4,5 and 6$)$. This could be due to the fact that the asymmetry of the task makes the syntactic cue more difficult to follow, since participants in the reliable-syntax condition have to create homophones in order to follow the cue. It is also possible that the familiar named objects or actions being shown on the screen are very attractive and hard to ignore. We discuss this finding more in the Discussion section.

\section{Eyetracking}

Overall proportion of looks. There was a main effect of condition $(\beta=0.32, \mathrm{SE}=0.06, \mathrm{t}$ $=5.48, \mathrm{p}<.0001)$ : participants in the reliable-syntax condition looked more at the syntactic choice than participants in the reliable-semantics condition. There was also a main effect of age $(\beta=0.18, \mathrm{SE}=0.06, \mathrm{t}=3.21, \mathrm{p}=.002)$ : children were looking at the syntactic choice more than adults. There was also a significant interaction between age and condition $(\beta=-0.26$, SE $=.08, \mathrm{t}=-3.34, \mathrm{p}=.001)$ : When analyzing children and adults separately, an effect of condition was found for adults only (adults: $\beta=0.31, \mathrm{SE}=0.07, \mathrm{t}=4.75, \mathrm{p}<.0001$; children: $\beta=0.06$, $\mathrm{SE}=0.05, \mathrm{t}=1.36, \mathrm{p}=.18)$ : only adults looked significantly more to the syntactic choice in the reliable-syntax condition than in the reliable-semantics condition (Fig. 5). To test for a possible bias towards the semantic vs syntactic choice, we compared average looks to chance (50\%) using a one-sample $\mathrm{t}$ test, in all conditions taken together. A preference is observed towards semantics $(\mathrm{t}(83)=2.84, \mathrm{p}=0.006)$. 


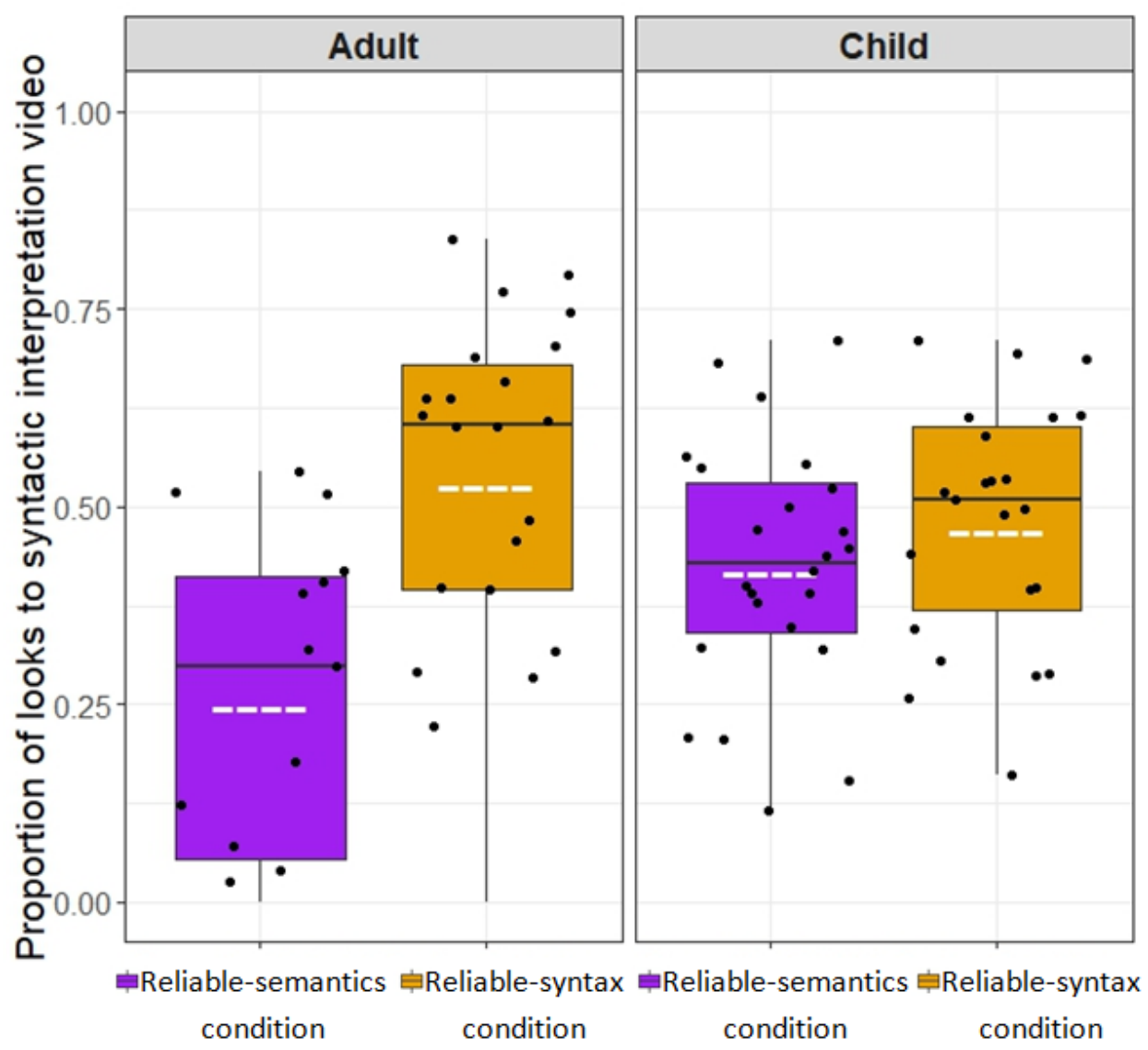

Figure 5. Mean proportion of looks towards syntactic choice per condition (reliable-semantics vs reliable-syntax) for adults and children. Black dots represent individual participants. The lower and upper hinges correspond to the first and third quartiles, the dotted white lines represent the means, and the black lines within the squares represent the median. The top and bottom whiskers denote the minimum/maximum value. As can be seen, adults in the reliable-syntax condition look more to the syntactic interpretation video than those in the reliable-semantics condition. However, there is no significant difference for children.

Time-course analysis. For adults only, a time-window was identified in which the proportion of looking times towards the syntactic choice differed significantly between conditions: between 4020ms and 6720ms, just after the first content word onset, adults looked significantly more towards the syntactic choice in the reliable-syntax condition than in reliablesemantics condition $(\mathrm{p}=.002)$. No such time window was identified for children (see Fig. 6). 


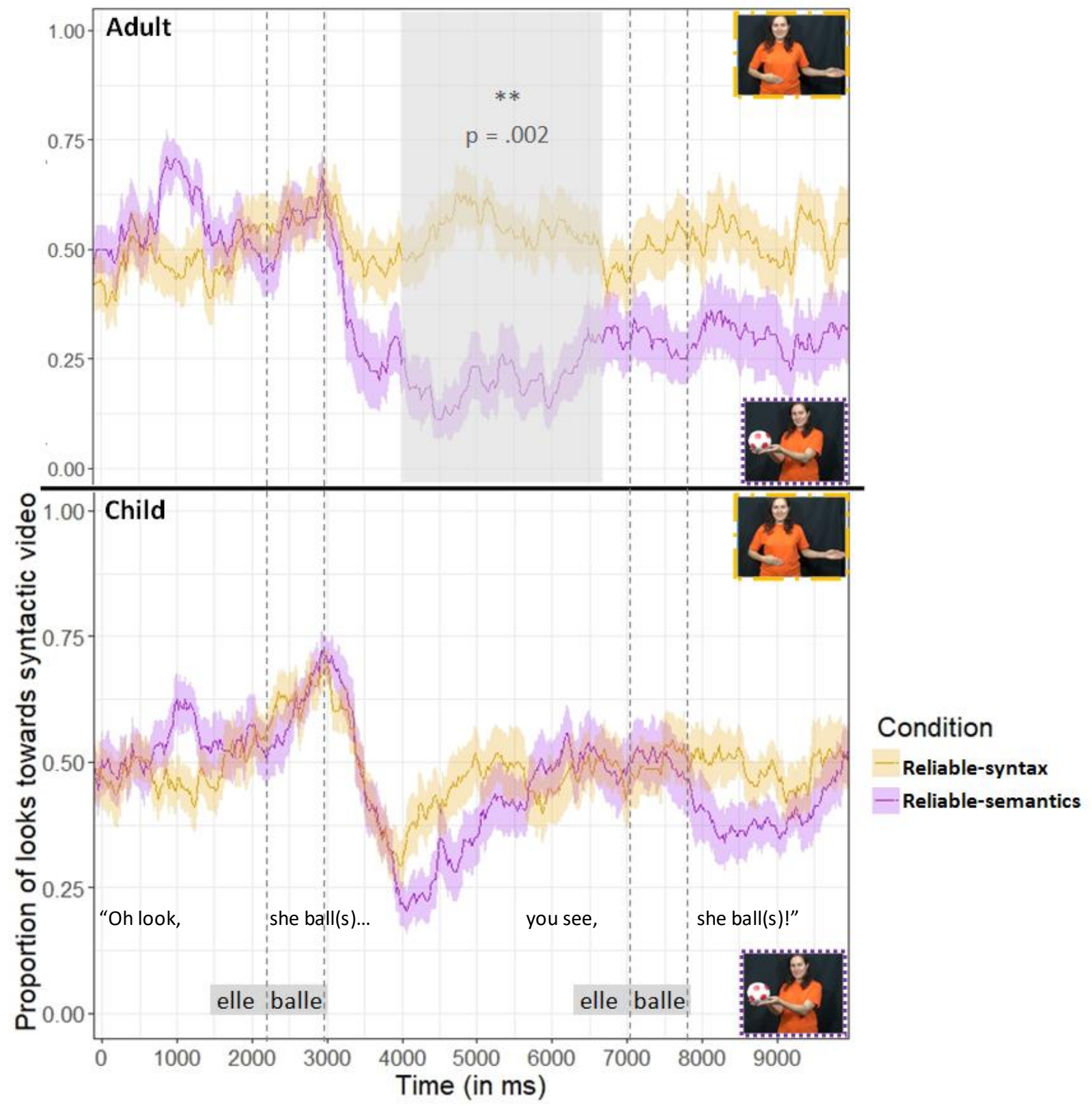

Figure 6. Proportion of looks towards syntactic choice, as a function of time, per condition (reliablesemantics/reliable-syntax) for adults (top half of the figure) and for children (bottom half of the figure).

Fig. 6 shows that the general gaze behavior is consistent with the presented conflict situation: When the function word appears (end of pronoun or article from $2.4 \mathrm{~s}$ ), the mean proportion of looks towards the syntactic choice increases (i.e. peak up towards the syntactic choice), suggesting that both adults and children follow the syntactic cue; when the content word appears there is an increase of looks to the semantic choice, the thing or action that is named (i.e. peak 
down towards the semantic choice from 3s). However, this second semantic peak is visually smaller in the reliable-syntax condition than in the reliable-semantics condition (and is statistically significant in adults only), which is consistent with the pointing results. Even though there is no significant difference between the groups in children, their gaze behavior is consistent with the children synchronically following the different cues as they unfold, and then hesitating between the two interpretations when the sentence is complete. 


\section{Discussion}

The noisy channel account of language comprehension states that listeners interpret sentences by weighting the reliability of different cues in context (Gibson, et al., 2013; for an earlier suggestion integrating cue validity and language acquisition, see Bates \& MacWhinney, 1989). Indeed, four-to-five-year-old children have been shown to resolve ambiguities by prioritizing the more reliable source of information over the less reliable one (Yurovsky et al., 2017). Here we tested whether the noisy channel account can also explain a phenomenon in word learning, namely: How do children know when to downplay or adjust their syntactic expectations, and when to rely on them to infer the meaning of a novel word? In the induction phase, we added noise to children's syntactic signal by using videos that made them understand the speaker was making grammatical errors. A second group of children heard the same sentences, but was led to think that the same sentences were grammatical sentences introducing a novel word. At test, sentences were put in conflict between children's preexisting semantic and syntactic knowledge in order to test whether children adapted their prior knowledge dynamically by weighting the reliability of these different sources of information.

We hypothesized that children will adapt to the reliability of different sources of information in their input to prioritize the most reliable source of information when there is a conflict. Thus, children who were exposed to reliable syntax will rely on syntax more than children who heard a speaker repeatedly make syntactic errors. This hypothesis was verified by our pointing results, showing more pointing towards the syntactic choice in the reliable-syntax condition relative to the reliable-semantics condition. Both children and adults tended to interpret sentences towards the syntactically reasonable choice more when syntax was always reliable, than when semantics was always reliable. These results show that both children and adults are able to use the relative reliability of syntactic and semantic information to resolve a conflict between them. The integration of these sources of information is done by evaluating 
the relevance of each type of information and adapting their weight in context. This first extends the results of Yurovsky et al. (2017), to the question of adaptability of syntactic and semantic sources of information (Yurovsky and colleagues focused on pragmatic vs. phonological information) in language processing. In addition, in our task, children not only used their knowledge of language to comprehend ambiguous sentences, as in Yurovsky et al., they also tended to accept novel meanings for familiar word forms when syntax was reliable but less so when it was not. Our results imply that adaptation could be an active mechanism in language learning.

In language learning, research has shown that children can adapt their syntactic representations in context by relying on the words they know (Havron, de Carvalho, Fiévet \& Christophe, 2019), and that children can adapt their semantic representations, inferring that a word-form they know refers to a new semantic entity (learn a homophone, Dautriche, Fibla, Fiévet, \& Christophe, 2018). However, until now it was unclear how children know which of these representations to change when they are in conflict. We have shown that children are able to adjust the weight of different sources of information to prioritize between them, and adapt or downplay their expectations accordingly.

Could it be, however, that during the induction phase participants simply learned that the correct answer corresponds to novel vs. familiar meanings depicted in the videos and use that strategy in the test phase, without paying attention to how reliably the speaker is using syntax vs semantics? We think it is unlikely that our participants could adopt this strategy to complete our task successfully, for three main reasons. First of all, participants are not given feedback for their responses, so they are concluding for themselves what is the correct response (note that this is true for the interleaved test trials as well as for induction trials). Second, such a strategy would require high levels of metalinguistic awareness (thinking of something as a “familiar object/action" vs. "novel object/action"), an ability which is highly underdeveloped 
in children this age (see e.g., Havron \& Arnon, 2017; Olson, 1996). What makes such a rule even harder to detect explicitly is that before the first test item appears, the participants had only two opportunities to make a choice that includes a novel item (for the reliable-syntax group) or a grammatical error (for the reliable-semantics group). The same participant has had more opportunities to learn the regularities in the reliability of the speaker's use of syntax or semantics, because they have already experienced six total induction items containing information about semantic or syntactic noise (as they have had four single-video induction items and two two-video induction items). Even for adults, who do have the required metalinguistic ability, we find evidence that this is not the case that such an explicit rule was formed. As we describe below in more details, as well as in our exploratory items and results presented in the supplementary materials, adults seem to have not only learned to ignore syntax in the reliable-semantics condition, but to actually learn new contingencies - that articles predict verbs and pronouns predict nouns. They could not have done this if they were relying on the proposed metalinguistic strategy to always look at familiar or novel words. A final reason why we believe children are not following an explicit rule they have formed and ignored the speaker's reliability is their gaze behavior. As described in more detail below, children shift their gaze as the sentence unfolds, synchronically following the different cues before hesitating between both interpretations. An explicit strategy to choose or ignore the novel word or the semantic congruency would enable children to look directly at their prechosen alternative and ignore the sentence.

While both age groups showed an effect of the manipulation, we found that this effect was stronger for adults than for children. According to error-driven learning models, children are predicted to adjust more than adults to violations of syntactic expectations, as their syntactic representations are less robust than adults' (e.g., Peter, Chang, Pine, Blything, \& Rowland, 2015). However, previous research has also shown smaller adaptation effects in children than 
in adults (Havron, de Carvalho, Fiévet, \& Christophe, 2019), possibly due to their slower cognitive processing and lesser likelihood of integrating the induction sentences (see also Bates \& MacWhinney, 1989). In our study as well, adults might have been able to integrate the induction phase more thoroughly, understand and integrate the novel meanings for the homophones, and/or better realize the scope of the syntactic violations. Another way in which task demands might impact children and adults differently is the amount of noise in our measure: adults tend to focus all their attention on the task, and perform it as error-free as they are able; children are typically less focused, and even when the task is relatively easy for them, there are always more mistakes. As a result, children's performance may move towards the center, resulting in smaller observed effects. Due to the added noise when testing children, and to the fact that task demands likely weight more strongly on their cognitive resources, we cannot conclude from our results that children adapt less to the reliability of different sources of information than adults do. To be able to conclude this, researchers would have to design a task where noise or task demands are equal for both age groups. Until then, the question of whether children adapt less than adults is open for debate. That being said, multiple recent studies did find weaker prediction or prediction adaptation effects the younger the participants (Havron, Babineau, \& Christophe, under revision; Gambi, Gorrie, Pickering, \& Rabagliati, 2018; Havron et al., 2019), while these studies did not solve the problem of experimental noise and task demands, these findings lead us to at least consider the possibility that adults are indeed quicker to adapt.

In addition to testing children and adults' explicit choice, we measured participants' gaze behavior. Gaze data confirmed this adaptation process for adults, showing that they looked more towards the syntactic choice in the reliable-syntax condition than in the reliable-semantics condition. These results were not statistically significant for children. However, their gaze behavior helps to verify that the conflict situation between the two sources of information is 
indeed taken into account by the participants, both adults and children. In adults, both groups look more at the syntactic choice right after the syntactic cue. Participants in the reliable-syntax condition continue to look at the syntactic choice when the content word appears, ignoring the original meaning of the content word, while participants in the reliable-semantics condition switch to look at the semantic choice after the familiar word is pronounced, and stay with their choice. In children, we seem to see an even clearer orientation change, from the syntactic to the semantic choice as the sentence unfolds, but there is no significant difference between the groups. This suggests that children spotted each of the cues, and that they were trying to integrate these conflicting cues as the sentence unfolded. Though not significantly so, children in the reliable-syntax condition seem to recover from the semantic cue a bit faster. This tendency is encouraging for further research with gaze behavior.

Additionally, we observed a general semantic preference in conflict situations: children's results are shifted to the semantic choice, and adults' results in the reliable-semantics condition are at $100 \%$ of semantic choice, unlike results in the reliable-syntax condition, which are not at $100 \%$ of syntactic choice. Bates and MacWhinney (1989) discuss the fact that children have a tendency to rely more on semantic information, and only around the age of six do they develop an adult-like reliance in syntax. In our experiment a semantic preference was found for both age groups. However, this result could also be due to a task asymmetry: In addition to ignoring the familiar meaning of the word, participants in the reliable-syntax condition have another step to perform compared to the reliable-semantics condition - create homophones to be able to follow the syntax. The task might thus be more difficult. Moreover, the linearity of language makes one cue appear before the other; syntax could be better retained because the function word is heard first, but semantics could be better retained because of its recency. Indeed, the timeline of gaze shows a change towards the semantic choice at the onset of the semantic cue (the content word), and then the gaze seems to have difficulty breaking off from it to go back 
to balance, independently of the condition. Thus, with eyetracking measures, we can see that disregarding the familiar meaning by creating a homophone might be a difficult task, since the semantic information seems to attract more attention than the syntactic choice.

This study has three main limitations. First, we tested four-to-five-year-old children, and not younger children. It is possible that the mechanism of adapting to the reliability of different sources of information is active in children from the very first stages of language acquisition, and partly explains how infants manage to decide when syntactic representations need to be adjusted, and when lexical items need to be adjusted. To test this hypothesis, future studies should test younger children, at the beginning stages of language acquisition, with a different paradigm than the one we used, one more appropriate for younger ages (but see Havron, Babineau \& Christophe, under review, for failure to show adaptation in 18-month-old infants). Second, future studies should test whether participants who followed the semantic cue (and chose the video corresponding to the familiar word) only learned to ignore syntax, or whether they actually adapted their syntactic expectations to contradict a lifetime of experience in the context of the study - since our study was not able to answer this question. We had two exploratory items where the options presented to children are a novel object and a novel action (and the familiar action or object being named is not present) added at the end of our long experiment, which we describe in the supplementary materials. These two items were not enough to systematically explore whether children in the reliable-semantics condition have been ignoring syntax, or whether they have actually learned predictions that go against their life-long experience, and indeed we found no difference between the two groups for children. Adults, however, did show a difference between groups, with adults in the reliable-semantics group expecting pronouns to precede nouns and determiners to precede verbs, the opposite of their life-long expectations. Since the current experiment found stronger adaptation effects for adults than children, it is possible that given a more systematic exploration, with more test items 
and greater statistical power, we would find that children are also able to change their syntactic expectations. The inherent asymmetry between our two experimental conditions in what gets learned is another limitation to our ability to make broad generalizations relating to language acquisition. Since we have no control condition, we do not know if the default choice for children with no induction phase would have been to learn a novel homophone (as in the reliable-syntax condition) or to ignore/adapt syntactic expectations (as in the reliable-semantics condition). If the default is to learn a novel homophone, and children in the reliable-semantics condition are only learning to ignore syntax and listen for the content word, then there is not much learning going on in our experiment, and our results say more about language processing than its acquisition. However, we deem this option unlikely due to the general preference we found for the semantic interpretation. Thus, we believe it more likely that children and adults increased the weight of syntax to learn novel homophones in one condition, and either abandoned or adjusted their syntactic expectations in the second condition.

To conclude, the current study supported the noisy channel hypothesis (Gibson et al., 2013; Yurovsky et al., 2017), extending it to local adaptation to syntactic and semantic information sources. Moreover, it showed that this hypothesis could also be valid for learning situations, i.e. when novel words are acquired: Children are able to adapt to a context and to integrate linguistic sources of information by using these sources' reliability. They interpret sentences containing novel information in a particular context - here, as described above - in the reliable-syntax condition, they infer that a familiar word form is used in a novel way, and interpret the sentence as containing a homophone. The greater use of some sources of information depending on their reliability in the situation shows that the weight of different sources of information can change with the context (Federmeier, 2007) even for children. We also find stronger effects in adults, which can either be due to task demands or to greater flexibility in predictions in adults (Huettig, 2015). 


\section{References}

Bates, E., \& MacWhinney, B. (1989). Functionalism and the Competition Model. In The crosslinguistic study of sentence processing. (pp. 3-76). New York: Cambridge University Press.

Bates, D., Maechler, M., Bolker, B., \& Walker, S. (2015). Fitting Linear Mixed-Effects Models Using lme4. Journal of Statistical Software, 67(1), 1-48. doi:10.18637/jss.v067.i01.

Bernal, S., Lidz, J., Millotte, S., \& Christophe, A. (2007). Syntax Constrains the Acquisition of Verb Meaning. Language Learning and Development, 3(33), 325-341. https://doi.org/10.1080/15475440701542609

Brusini, P., Dehaene-Lambertz, G., van Heugten, M., de Carvalho, A., Goffinet, F., Fiévet, A. C., \& Christophe, A. (2017). Ambiguous function words do not prevent 18-month-olds from building accurate syntactic category expectations: An ERP study. Neuropsychologia, 98, 4-12. https://doi.org/10.1016/j.neuropsychologia.2016.08.015

Cauvet, E., Limissuri, R., Millotte, S., Skoruppa, K., Cabrol, D., \& Christophe, A. (2013). Function Words Constrain On-Line Recognition of Verbs and Nouns in French 18-MonthOlds. Language Learning and Development, 10(1), 1-18. https://doi.org/10.1080/15475441.2012.757970

Chan, A., Lieven, E., \& Tomasello, M. (2009). Children's understanding of the agent-patient relations in the transitive construction: Cross-linguistic comparisons between Cantonese, German, and English. Cognitive Linguistics, 20(2), 267-300.

Dautriche, I., Fibla, L., Fiévet, A.-C. \& Christophe, A. (2018). Learning homophones in context: Easy cases are favored in the lexicon of natural languages, Cognitive Psychology, 104, 83105.

Dautriche, I., Swingley, D., \& Christophe, A. (2015). Learning novel phonological neighbors: Syntactic category matters. Cognition, 143, 77-86. https://doi.org/10.1016/j.cognition.2015.06.003

Dink, J., \& Ferguson, B. (2018). eyetrackingR. R package version 0.1.8, http://www.eyetrackingR.com.

Federmeier, K. D. (2007). Thinking ahead: The role and roots of prediction in language comprehension, Psychophysiology, 44(4), 491-505. https://doi.org/10.1111/j.1469$\underline{8986.2007 .00531 . \mathrm{X}}$

Frank, M. C., Braginsky, M., Yurovsky, D., \& Marchman, V. A. (2017). Wordbank: An open 
repository for developmental vocabulary data. Journal of child language, 44(3), 677694.doi: 10.1017/S0305000916000209.

Gambi, C., Gorrie, F., Pickering, M. J., \& Rabagliati, H. (2018). The development of linguistic prediction: Predictions of sound and meaning in 2- to 5-year-olds. Journal of Experimental Child Psychology, 173, 351-370. https://doi.org/10.1016/j.jecp.2018.04.012

Gelman, A., Jakulin, A., Pittau, M. G., \& Su, Y. S. (2008). A weakly informative default prior distribution for logistic and other regression models. Annals of Applied Statistics, 2(4), 1360-1383. https://doi.org/10.1214/08-AOAS191

Gibson, E., Bergen, L., \& Piantadosi, S. T. (2013). Rational integration of noisy evidence and prior semantic expectations in sentence interpretation. Proceedings of the National Academy of Sciences of the United States of America, 110(20), 8051-6. https://doi.org/10.1073/pnas.1216438110

Gleitman, L. (1990). The Structural Sources of Verb Meanings. Language Acquisition, 1(1), 355.

Havron, N., \& Arnon, I. (2017). Minding the gaps: literacy enhances lexical segmentation in children learning to read. Journal of Child Language, 44(6), 1516-1538. Retrieved from doi:10.1017/S0305000916000623

Havron, N., Babineau, M. \& Christophe, A. (in principle accepted registered report under stage 2 revision). 18-month-olds fail to use recent experience to infer the syntactic category of novel words. Developmental Science.

Havron, N., de Carvalho, A., Fiévet, A., \& Christophe, A. (2019). Three- to Four-Year-Old Children Rapidly Adapt Their Predictions and Use Them to Learn Novel Word Meanings. Child Development, 90(1), 82-90. https://doi.org/10.1111/cdev.13113

Huettig, F. (2015). Four central questions about prediction in language processing. Brain Research, 1626, 118-135. https://doi.org/10.1016/j.brainres.2015.02.014

Jaeger, T. F. (2008). Categorical Data Analysis: Away from ANOVAs (transformation or not) and towards Logit Mixed Models. Journal of Memory and Language, 59(4), 434-446. https://doi.org/10.1016/j.jml.2007.11.007.Categorical

Kaschak, M. P. (2006). What this construction needs is generalized. Memory \& Cognition, 34(2), 368-379. https://doi.org/10.3758/BF03193414

Kedar, Y., Casasola, M., Lust, B., \& Parmet, Y. (2017). Little Words, Big Impact: Determiners 
Begin to Bootstrap Reference by 12 Months. Language Learning and Development, 13(3), 317-334. https://doi.org/10.1080/15475441.2017.1283229

Markman, E. M., \& Wachtel, G. F. (1988). Children's use of mutual exclusivity to constrain the meanings of words. Cognitive Psychology, 20(2), 121-157. https://doi.org/10.1016/00100285(88)90017-5

New, B., Pallier C., Ferrand L., Matos R. (2001) Une base de données lexicales du français contemporain sur internet: LEXIQUE, L'Année Psychologique, 101, 447-462. http://www.lexique.org

Olson, D. R. (1996). Towards a psychology of literacy: On the relations between speech and writing. Cognition, 60(1), 83-104.

Peter, M., Chang, F., Pine, J. M., Blything, R., \& Rowland, C. F. (2015). When and how do children develop knowledge of verb argument structure? Evidence from verb bias effects in a structural priming task. Journal of Memory and Language, 81(December), 1-15. https://doi.org/10.1016/j.jml.2014.12.002

Piantadosi, S. T., Tily, H., \& Gibson, E. (2012). The communicative function of ambiguity in language. Cognition, 122(3), 280-291. https://doi.org/10.1016/j.cognition.2011.10.004

Rabagliati, H., Pylkkänen, L., \& Marcus, G. (2012). Top-down influence in young children's linguistic ambiguity resolution. Developmental Psychology, 49(6), 1076-1089. https://doi.org/10.1037/a0026918

Shi, R., \& Melançon, A. (2010). Syntactic Categorization in French-Learning Infants. Infancy, 15(5), 517-533. https://doi.org/10.1111/j.1532-7078.2009.00022.x

van Heugten, M. \& Christophe, A. (2014). Learning to accommodate syntactic violations during online speech perception. International Conference on Infant Studies, Berlin, 3-7 Juillet 2014

Waxman, S., Lidz, J., Braun, I. E., \& Lavin, T. (2009). 24-Month-Old Infants Interpretations of Novel Verbs and Nouns in Dynamic Scenes. Cognitive Psychology, 59(1), 67-95. https://doi.org/10.1016/j.cogpsych.2009.02.001.24-Month-Old

Wickham, H. (2016) ggplot2: Elegant Graphics for Data Analysis. Springer-Verlag New York,

Yurovsky, D., Case, S., \& Frank, M. C. (2017). Preschoolers flexibly adapt to linguistic input in a noisy channel. Psychological science, 28(1), 132-140. https://doi.org/10.1177/0956797616668557 


\section{Appendix: Stimuli}

\begin{tabular}{|c|c|c|c|}
\hline Sentence heard & English translation & Video 1 & Video 2 \\
\hline Une voiture & A car & A girl who draws & A car \\
\hline Elle danse & She dances & A girl & A nipple \\
\hline Elle dessine & She draws & A girl & \\
\hline Une tétine & A pacifier & A girl who dances & $\mathrm{A}_{1}$ \\
\hline
\end{tabular}

Table A1. Warm-up items, for both conditions randomly chosen between voiture + danse (car + dance) and dessine + tétine (draw + pacifier). These are two items without conflict, one familiar noun and one familiar verb.

\begin{tabular}{|c|c|c|c|}
\hline Sentence heard & English translation & Semantic video & Distractor \\
\hline \multirow{5}{*}{ Elle robe } & One-video induction: & \\
\hline & & & \\
& & & \\
& & & \\
& & & \\
Elle fraise & She strawberrys & A strawberry & No distractor \\
\hline
\end{tabular}




\begin{tabular}{|c|c|c|c|}
\hline Une parle & A speaks & A girl who speaks & No distractor \\
\hline Une tourne & A turns & A girl who turns & No distractor \\
\hline \multicolumn{4}{|c|}{ Induction: } \\
\hline Elle pomme & She apples & An apple & A girl who reads \\
\hline Elle chaise & She chairs & A chair & A girl who reads \\
\hline Une mange & An eats & A girl who eats & A shoe \\
\hline Une tousse & A caughs & A girl who caughs & A hat \\
\hline
\end{tabular}

Table A2. Induction items in reliable-semantics condition (four familiar nouns and four familiar verbs) 


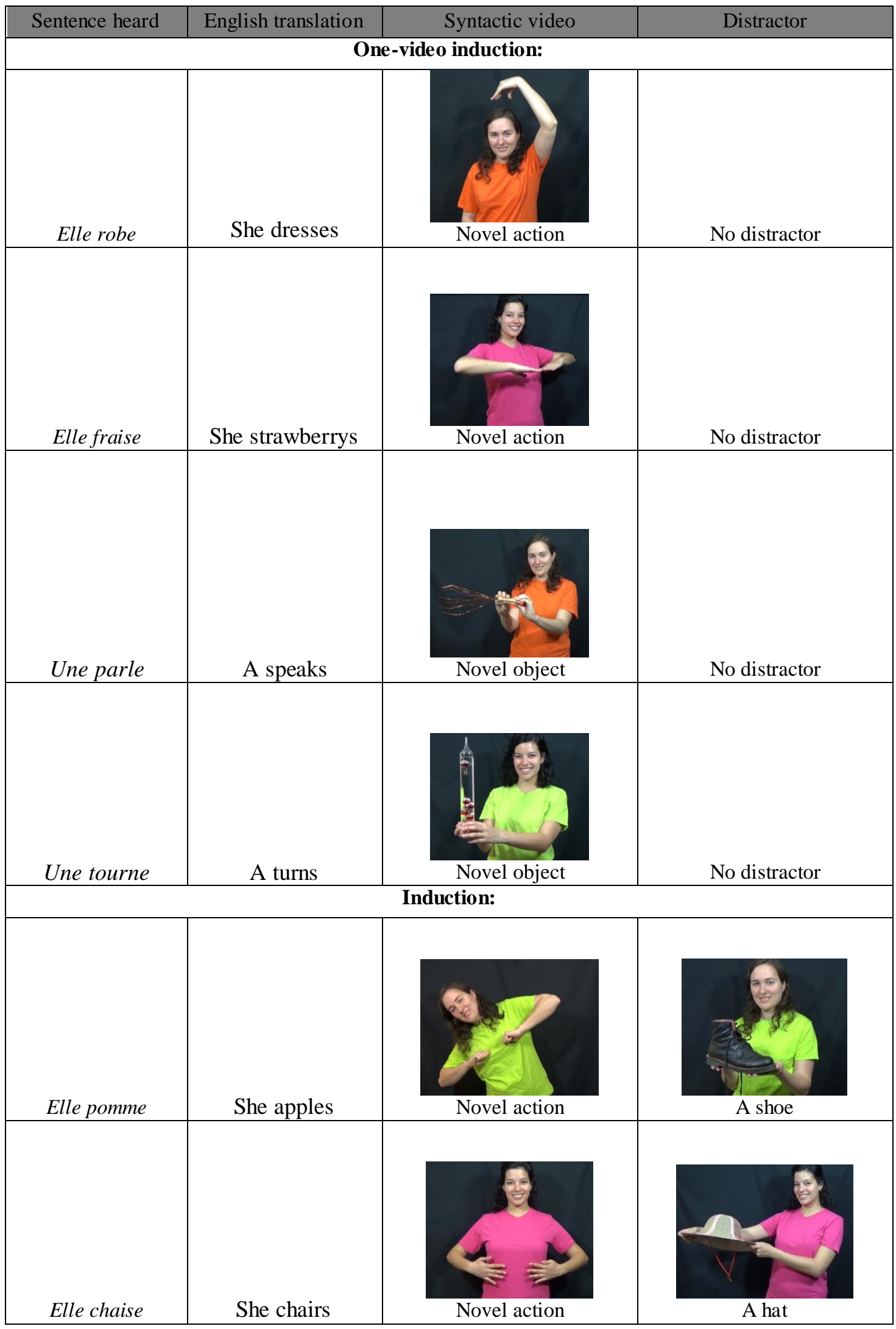




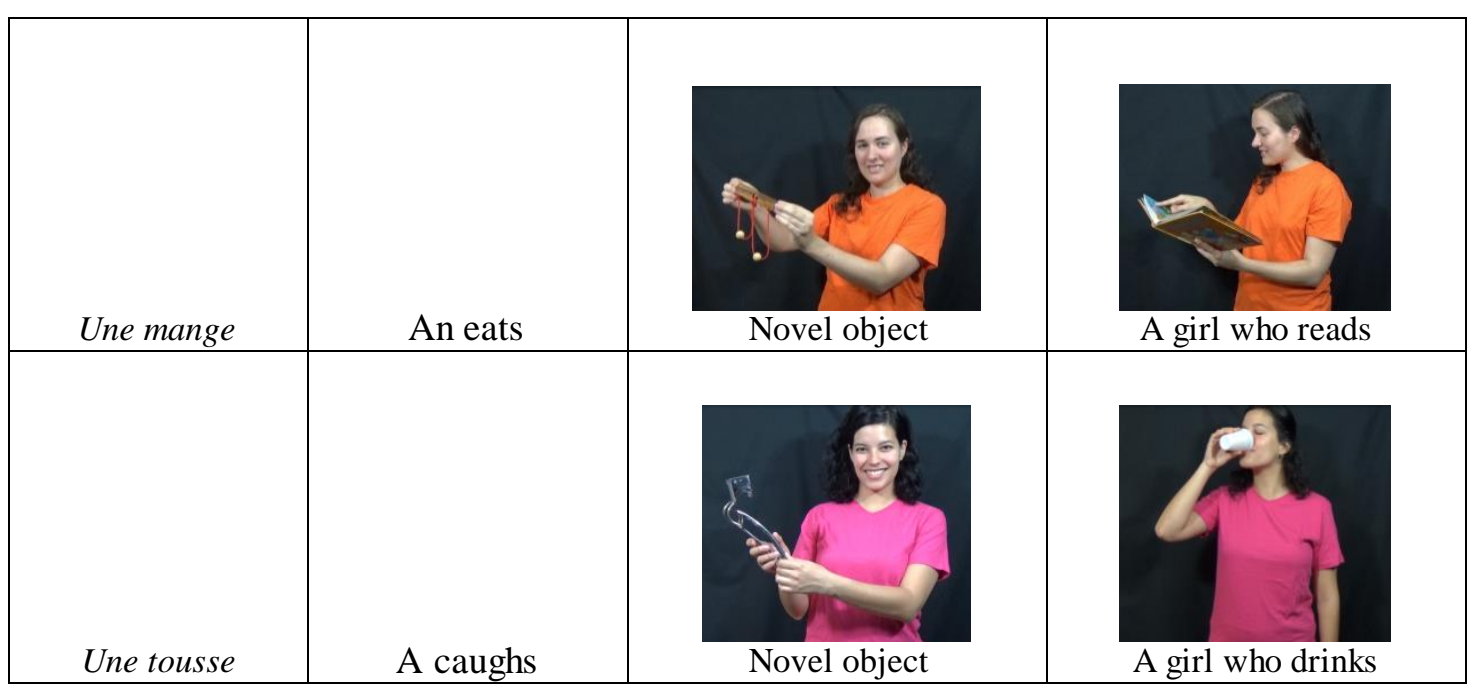

Table A3. Induction items in reliable-syntax condition (four familiar nouns and four familiar verbs) 


\begin{tabular}{|c|c|c|c|}
\hline Sentence heard & English translation & Video 1 & Video 2 \\
\hline Elle balle & She balls & A ball & Jovel action \\
\hline Elle lune & She moons & A moon & Jovel action \\
\hline Une saute & A jumps & A girl who jumps & Jovel object \\
\hline Une dort & A sleeps & A girl who sleeps & ect \\
\hline
\end{tabular}

Table A4. Conflict-test items, the same for both conditions (two familiar nouns and two familiar verbs) 


\begin{tabular}{|c|c|c|c|}
\hline Sentence heard & English translation & Video 1 & Video 2 \\
\hline $\begin{array}{c}\text { Elle nuve / une } \\
\text { nuve }\end{array}$ & She nuves/a nuves & Novel object & Novel action \\
\hline & & & \\
$\begin{array}{c}\text { Une dase / elle } \\
\text { dase }\end{array}$ & She dases/a dases & Novel action & Novel object \\
\hline
\end{tabular}

Table A5. Novel-word test items, the same for both conditions (two novel words, one used as a noun and one as a verb) - these items are described in the supplementary materials. 